\title{
Modulation Transfer Function Analysis in Myopic Model Eye
}

\author{
Shah Farez Othman ${ }^{1,2,3,4^{*}}$, Nizam Tamchek ${ }^{2}$, Farah Diana Muhammad ${ }^{2}$ and Mohd Hafidz Ithnin ${ }^{1}$ \\ ${ }^{1}$ Department of Optometry \& Visual Sciences, Kulliyyah of Allied Health Sciences, International Islamic University \\ Malaysia, Jalan Sultan Ahmad Shah, Bandar Indera Mahkota, 25200 Kuantan, Pahang, Malaysia \\ ${ }^{2}$ Department of Physics, Faculty of Science, Universiti Putra Malaysia, 43400 UPM Serdang, Selangor, Malaysia \\ ${ }^{3}$ SEGi EyeCare, SEGi University, No. 9, Jalan Teknologi, Taman Sains Selangor, Kota Damansara, PJU 5, 47810 \\ Petaling Jaya, Selangor, Malaysia \\ ${ }^{4}$ School of Optometry, Faculty of Medicine \& Health Sciences, UCSI University, No. 1, Jalan Menara Gading, UCSI \\ Heights (Taman Connaught), 56000 Cheras, Kuala Lumpur, Wilayah Persekutuan, Malaysia
}

\begin{abstract}
Hitherto, the eye modelling is based on the emmetropic eye taken its ocular optical components value from the population-based studies. However, no studies have been done to study the effect of aberration of myopic refractive error by modelling the eye using the parameters from ocular biometrics and ray tracing method. This study aimed to determine the modulation transfer function (MTF) of myopic refractive error using eye modelling and ray tracing technique. Three eye models had been successfully modelled in Zemax software, namely, emmetropic Liou and Brennan, myopic Liou and Brennan, corrected myopic Liou and Brennan. The optical performance of the eye models were tested using the MTF. From the MTF analysis at 100 cycles/mm, the MTF value of both tangential and sagittal rays for myopic Liou and Brennan eye was the lowest compared to its emmetropic model. Also, the MTF value of the corrected myopic Liou and Brennan model was higher compared to the uncorrected myopic model. However, the corrected myopic model produced lower MTF values for both tangential and sagittal MTF compared with the emmetropic model of Liou and Brennan. In this study, the accuracy of the MTF for myopia correction and emmetropia were calculated. It was found that the accuracy of the MTF value for corrected myopia at tangential and sagittal rays was lower.
\end{abstract}

Keywords: model eye; modulation transfer function; myopia; optical performance

\section{INTRODUCTION}

The vision that is produced by the human eye is a result from light impinges through the complex built in of ocular optical components. Hence, the eye model (schematic) that is simpler than the real eye has been designed due to the complexity of the optics of a real eye (Thibos \& Bradley, 1999; Zapata-Diaz et al., 2019). Eye models are available with a range of complexity, but at minimum, they include numerical values for radii of curvature, distance between refracting surfaces, and indices of refraction.
Previous myopia studies have proven that myopic refractive error is associated with eyeball elongation, in particular, the vitreous chamber depth increment (Gwiazda et. al., 2002; Jiang \& Woessner, 1996; Sun et al., 2015). In routine optometric examination, clinical refraction has been regarded as the conventional way of obtaining myopic refractive error. These clinical refraction techniques, namely, autorefraction, retinoscopy and subjective refraction have been regarded as the standard procedures (Carlson, 2016; Elliot, 2014). Nevertheless, only few studies have been conducted to study the effect of aberration of myopic refractive error by modelling the eye using the parameters 
obtained from the ocular biometrics and ray tracing method (Hiraoka et. al., 2017; Liu \& Wang, 2019). Most of the eye modelling is based on emmetropic eye. Therefore, a question arises if we can develop a computer modelling of eye that can verify the axial type of myopia from the clinical refraction findings, using the numerical values of the parameters of ocular optical components and the fixed refractive indices of media from the existing chosen eye model. Also, the value of spectacle refractive error is used to correct the myopic eye model, and analyse its optical performance using the merit function in Zemax, i.e., the MTF analysis.

This study aim to assess the aberrations of the image quality of myopia, i.e., the modulation transfer function (MTF) using a ray tracing technique of an eye model. The image quality gathered from the study will be described and later a conclusion of the comparison of the proposed myopic eye can be deduced.

\section{MATERIALS AND METHOD}

The research followed the tenets of the Declaration of Helsinki, with the research approved by the Ethics Committee for Research Involving Human Subjects of Universiti Putra Malaysia (UPM) (FS-Jun 13(01) EXP) and with informed consent obtained from all subjects. The study cohort comprised 34 myopic subjects aged $21.80 \pm 2$ years, ranging from 18 to 36 years. Cycloplegic monocular spherocylinder refraction was performed on both eyes using a Jackson crossed-cylinder on a trial frame. Maximum plus and binocular balance to $\pm 0.25 \mathrm{D}$ were administered. The mean spherical equivalent refraction (SER) obtained from the clinical refraction technique was $-4.47 \pm 1.93 \mathrm{D}$, ranging from $-2.25 \mathrm{D}$ to $-9.75 \mathrm{D}$. This was measured at $15 \mathrm{~mm}$ vertex distance. The onset of myopia must be before the age of 16 years to ensure the myopia was axial in nature, i.e., the so called juvenile-onset myopia (Bullimore et. al., 1992; Chua et. al., 2018; Grosvenor \& Scott, 1991; McBrien \& Millodot, 1987; Zadnik \& Mutti, 2019). Subjects with greater than 0.75 D of astigmatism as measured by subjective refraction or with a corrected visual acuity poorer than $6 / 6$ in the test eye were excluded. Subjects were also excluded if they had any ocular disease in either eye, previous ocular surgery, or had intraocular pressure greater than $21 \mathrm{mmHg}$. Effective pupil diameter (EPD) measurements were taken with undilated pupils. Mean EPD was $2.59 \pm 0.43 \mathrm{~mm}$ with a range of $2-4$ $\mathrm{mm}$. The comparison phacometry measurement was conducted to determine the anterior and posterior crystalline lens curvatures. This was followed by keratometry to determine the anterior corneal curvature. Whereas, the Ascan ultrasound biometry technique was carried out to determine the parameters of anterior chamber depth, lens thickness and axial length.

The eye modelling was carried out using Zemax software (Zemax, 2012). The modelling is based on previous models of unaccommodated Liou and Brennan emmetropic eyes (Liou \& Brennan, 1997). Firstly, the validating of emmetropic eye using computer model utilising clinical ocular parameter from the population-based study was carried out (Liou \& Brennan, 1997). Secondly, the optical performance, i.e., the MTF was used in the validation process. Finally, a simple comparison will be proposed to make the validation process would be easily to be utilised in real application of correcting myopic problem.

The contrast of the gratings or known as modulation, $M$, in optical terms is defined as (Goss \& West, 2002)

$$
M=\frac{I_{\max }-I_{\min }}{I_{\max }+I_{\min }}
$$

whereby $M$ is the modulation, $I_{\max }$ and $I_{\min }$ are the maximum and minimum intensities respectively. The MTF is a plot of a reduction in modulation (contrast) when going from object to image, against a range of grating spacing (spatial frequency) in line pairs per degree or millimetre (Figure 1). For a diffraction-limited, i.e., aberration-free optical system, the spread function is relatively small due to the effects of diffraction. Thus, the decreasing modulation in the image is mainly because of smaller grating spacing. For optical system with significant aberrations or defocus, the spread function becomes wider and image contrast falls off more sharply. 


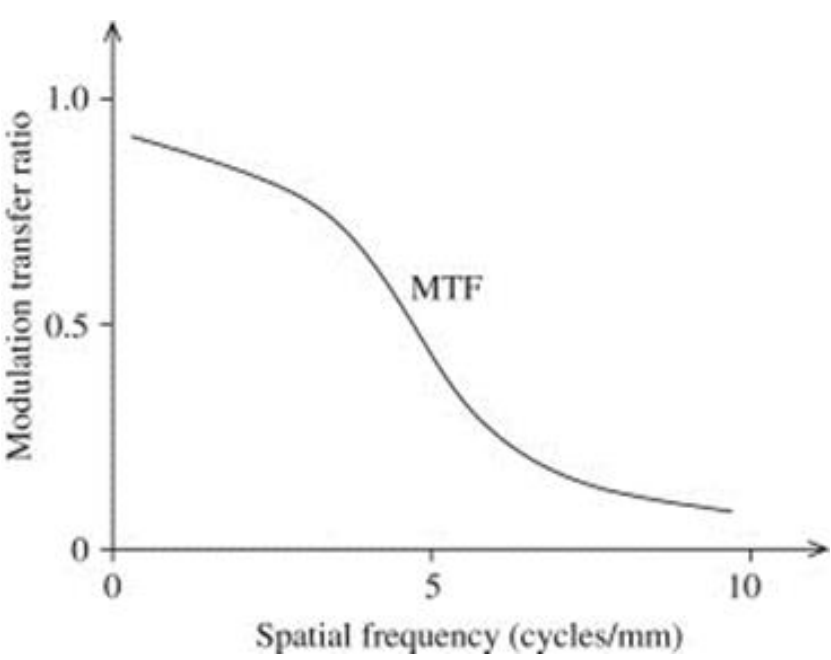

Figure 1. The Modulation Contrast function plots reduction in contrast between object and image for a range of spatial frequencies

The optical parameters of Liou and Brennan emmetropic and myopic eye models were entered in the Zemax's Lens Data Editor in the forms of rows and columns as shown in Table 1 and Table 2, respectively.

Table 1. Ocular parameters input of Liou and Brennan emmetropic eye model in lens editor of Zemax

\begin{tabular}{ccccc}
\hline $\begin{array}{c}\text { Sur- } \\
\text { face }\end{array}$ & $\begin{array}{c}\text { Radius } \\
(\mathbf{m m})\end{array}$ & $\begin{array}{c}\text { Thick- } \\
\text { ness } \\
(\mathbf{m m})\end{array}$ & $\begin{array}{c}\text { Refrac- } \\
\text { tive } \\
\text { Index }\end{array}$ & $\begin{array}{c}\text { Asphe- } \\
\text { ricity }\end{array}$ \\
\hline 1 & Infinity & 50.00 & - & 0.00 \\
2 & 7.77 & 0.50 & 1.376 & -0.18 \\
3 & 6.40 & 3.16 & 1.336 & -0.60 \\
4 & Infinity & 0.00 & 1.336 & 0.00 \\
5 & 12.40 & 1.59 & Grad A & -0.94 \\
6 & Infinity & 2.43 & Grad P & 0.00 \\
7 & -8.10 & 16.27 & 1.336 & 0.96 \\
8 & -12.00 & - & - & 0.00 \\
\hline
\end{tabular}

Table 2. Ocular parameters input of Liou and Brennan myopic eye model in lens editor of Zemax

\begin{tabular}{ccccc}
\hline $\begin{array}{c}\text { Sur- } \\
\text { face }\end{array}$ & $\begin{array}{c}\text { Radius } \\
\text { (mm) }\end{array}$ & $\begin{array}{c}\text { Thick- } \\
\text { ness } \\
\text { (mm) }\end{array}$ & $\begin{array}{c}\text { Refrac- } \\
\text { tive } \\
\text { Index }\end{array}$ & $\begin{array}{c}\text { Aspheri- } \\
\text { city }\end{array}$ \\
\hline 1 & Infinity & 50.00 & - & 0.00 \\
2 & 7.56 & 0.50 & 1.376 & -0.18 \\
3 & 6.40 & 3.40 & 1.336 & -0.60 \\
4 & Infinity & 0.00 & 1.336 & 0.00 \\
5 & 11.86 & 1.79 & Grad A & -0.94 \\
6 & Infinity & 2.00 & Grad P & 0.00 \\
7 & -7.26 & 16.98 & 1.336 & 0.96 \\
8 & -12.00 & - & - & 0.00 \\
\hline
\end{tabular}

An ophthalmic lens was designed by adding an eyeglass lens to the front of the Liou and Brennan myopic eye model. The lens power was taken as $-4.47 \mathrm{D}$ using the mean SER results calculated from the clinical refraction technique. This lens power was optimised to provide good imaging quality as the Liou and Brennan emmetropic model eye.

First, two surfaces between the Input Beam (Surface 1) and the Cornea (Surface 2) were inserted. These two surfaces represent the front and back surfaces of the ophthalmic lens. The ophthalmic lens was placed $15 \mathrm{~mm}$ from the eye. This 15 $\mathrm{mm}$ distance was chosen as a vertex distance from the anterior corneal apex to the back surface of the spectacle plane. The new surfaces, namely, Surface 2 and 3 are the front and back surfaces of the ophthalmic lens with a standard surface type. The lens had a thickness of $3 \mathrm{~mm}$ with a semidiameter (radius) of $20 \mathrm{~mm}$. The lens was selected as a polycarbonate material from the Glass Catalogue in Zemax (Zemax, 2012). The refractive index of the material is 1.58547 calculated at $581.1 \mathrm{~nm}$ wavelength using Sellmeier Equation as follows, i.e.:

$$
n^{2}-1=\frac{1.4182 \lambda^{2}}{\lambda^{2}-0.021304}
$$

whereby $n$ is the refractive index of the ophthalmic lens, and $\lambda$ is the light wavelength.

The equiconcave lens type was chosen for the myopia correction. The radius of curvatures for front and back surfaces were taken as $-\mathbf{2 6 1 . 9 6} \mathrm{mm}$ and $+261.96 \mathrm{~mm}$ obtained 
from the following power of Lens Maker's Equation (Tunnacliffe \& Hirst, 1996),

$$
F=\left(n_{p}-1\right)\left(\frac{1}{r_{1}}-\frac{1}{r_{2}}\right)
$$

where $\mathrm{F}$ is the power of the corrective lens used, $n_{p}$ is the refractive index of the lens, $r_{1}$ and $r_{2}$ are the radius of curvature of front and back surfaces, respectively. Table 3 summarise the Zemax Lens Data Editor using parameters of Liou and Brennan corrected myopic eye model.

Table 3. Ocular parameters input of Liou and Brennan corrected myopic eye model in lens editor of Zemax

\begin{tabular}{ccccc}
\hline $\begin{array}{c}\text { Sur- } \\
\text { face }\end{array}$ & $\begin{array}{c}\text { Radius } \\
\text { (mm) }\end{array}$ & $\begin{array}{c}\text { Thick- } \\
\text { ness } \\
\text { (mm) }\end{array}$ & $\begin{array}{c}\text { Refrac- } \\
\text { tive } \\
\text { Index }\end{array}$ & $\begin{array}{c}\text { Aspheri- } \\
\text { city }\end{array}$ \\
\hline 1 & Infinity & 50.00 & - & 0.00 \\
2 & -261.96 & 3.00 & 1.58547 & 0.00 \\
3 & +261.96 & 28.00 & - & 0.00 \\
4 & 7.56 & 0.50 & 1.37600 & -0.18 \\
5 & 6.40 & 3.40 & 1.33600 & -0.60 \\
6 & Infinity & 0.00 & 1.33600 & 0.00 \\
7 & 11.86 & 1.79 & Grad A & -0.94 \\
8 & Infinity & 2.00 & Grad P & 0.00 \\
9 & -7.26 & 16.98 & 1.33600 & 0.96 \\
10 & -12.00 & - & - & 0.00 \\
\hline
\end{tabular}

Finally, once the optical performances were achieved, the results were analysed and presented. The result from the modelling was then compared with each eye models. The finding from the comparison study was used to confirm on how optical performance of a myopic eye could be validated when it was in uncorrected and corrected status.

\section{RESULT AND DISCUSSION}

The 2-D layout of the emmetropic Liou and Brennan eye model from Zemax output is presented in Figure 2. The Liou and Brennan model refracting surfaces are shown accordingly starting from infinity to the anterior cornea, posterior cornea, anterior lens and posterior lens. According to Liou and Brennan model, the retinal curvature was taken into consideration for image formation (Smith et al., 2008).

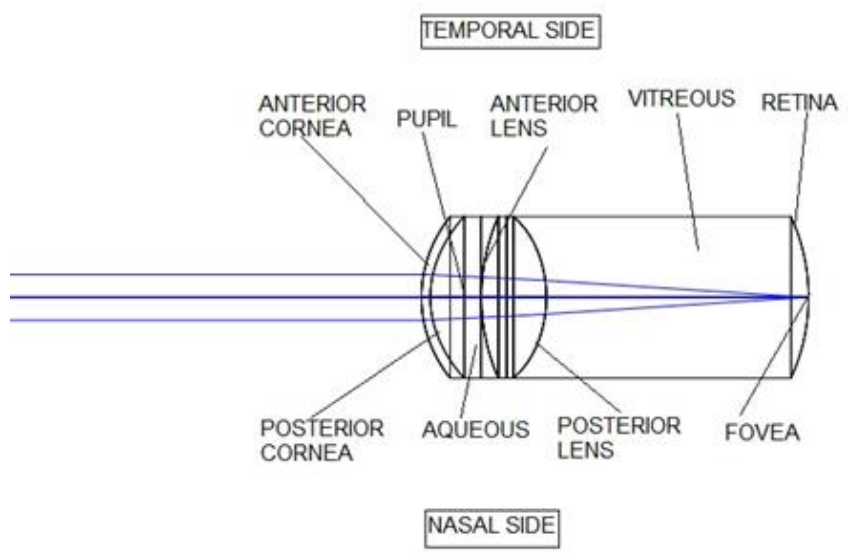

Figure 2. 2-D layout of emmetropic Liou and Brennan eye model

The tangential and sagittal MTF curves obtained for the emmetropic Liou and Brennan eye model at visible light wavelength ranging from $486.1 \mathrm{~nm}$ to $656.3 \mathrm{~nm}$ is presented in Figure 3. To illustrate the influence of decentration and tilt of the pupil, it was carried out by plotting the tangential and sagittal MTF.

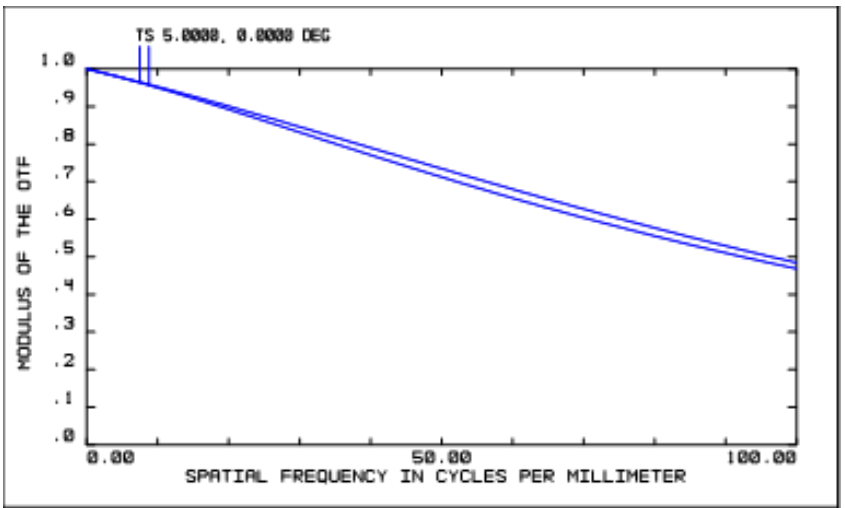

Figure 3. MTF for emmetropic Liou and Brennan eye models at light wavelength from $486.1 \mathrm{~nm}$ to $656.3 \mathrm{~nm}$. Tangential

ray is denoted by ' $\mathrm{T}$ ' and sagittal ray is denoted by ' $\mathrm{S}$ '.

From Figure 3, the MTF result for Liou and Brennan eye decreased almost in linear fashion along the increment of spatial frequency. At spatial frequency of $100 \mathrm{lp} / \mathrm{mm}$, the cutoff of MTF was 0.4842 and 0.4685 for tangential and sagittal, respectively. The MTF result also shows that both tangential and sagittal rays were not overlapping indicate that the model eye is elliptical in shape. It was due to that the Liou and Brennan eye having more refracting surfaces and taking into account the asphericity of each surface. The model also have 
distance between the surfaces and possess more than one refractive indices.

The 2-D layout of the myopic Liou and Brennan eye model from Zemax output is presented in Figure 4. The myopic Liou and Brennan model refracting surfaces are shown accordingly starting from infinity to the anterior cornea, posterior cornea, anterior lens and posterior lens.

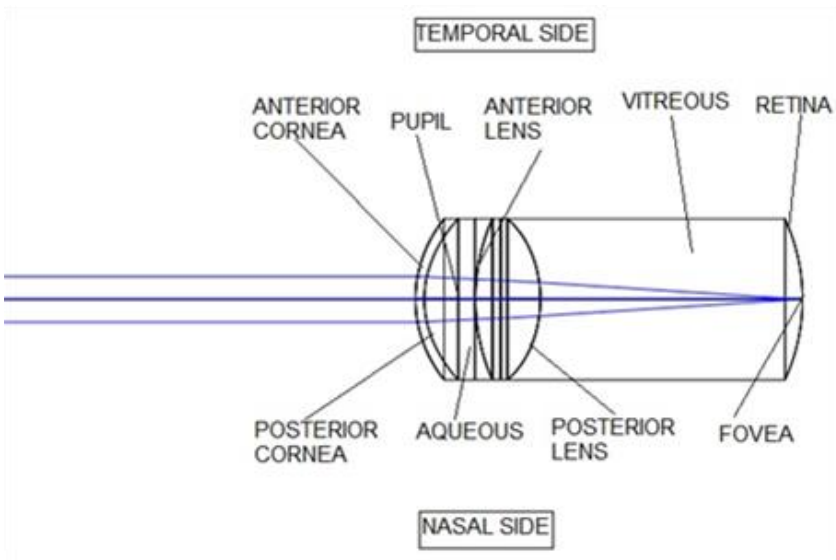

Figure 4. 2-D layout of myopic Liou and Brennan eye model

The tangential and sagittal MTF curves obtained for myopic Liou and Brennan eye model at visible light wavelength ranging from $486.1 \mathrm{~nm}$ to $656.3 \mathrm{~nm}$ are presented in Figure 5. For the myopic Liou and Brennan model, to illustrate the influence of decentration and tilt of the pupil, it was carried out by plotting the tangential and sagittal MTF.

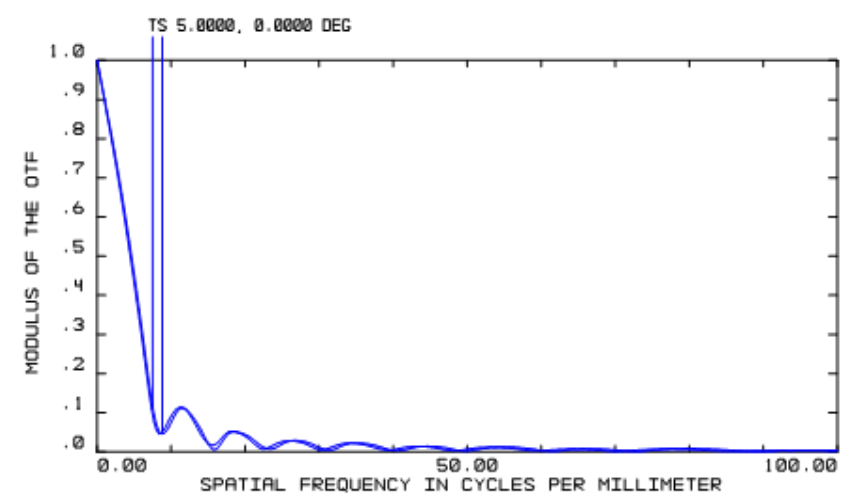

Figure 5. MTF for myopic Liou and Brennan eye model at light wavelength from $486.1 \mathrm{~nm}$ to $656.3 \mathrm{~nm}$. Tangential ray is denoted by ' $\mathrm{T}$ ' and sagittal ray is denoted by ' $\mathrm{S}$ '.

The MTF result for myopic Liou and Brennan model shown in Figure 5 is separated for tangential and sagittal rays. Also, it shows that the MTF starts from 1 (for perfect) and reduce to lower value with the increment of spatial frequency as the ray approach toward the posterior of the eyeball before reaching the retina.

At spatial frequency of $100 \mathrm{lp} / \mathrm{mm}$, the cut-off of MTF was 0.0035 and 0.0017 for tangential and sagittal, respectively. The MTF result also shows that both tangential and sagittal rays were not overlapping indicate that the model eye is elliptical in shape. It was due to the myopic Liou and Brennan eye having multiple refracting surfaces at different distances with different curvatures and refractive indices which caused the ray to be easily diffracted (Atchison \& Smith, 2000).

Further analysis on tangential and sagittal rays showed that the MTF of the myopic Liou and Brennan eye is lower compared to its emmetropic model. At $100 \mathrm{lp} / \mathrm{mm}$ spatial frequency, the MTF for tangential and sagittal ray were $0.35 \%$ and $0.17 \%$, respectively, which was lower than MTF value for emmetropic model, i.e., $48.42 \%$ and $46.85 \%$.

In contrast with emmetropic Liou and Brennan eye, the myopic model was modelled by replacing the refracting surfaces and distance between the refracting surfaces with the mean parameters of a myopic eye taken from the ocular biometry measurements. Overall, the tangential and sagittal ray degraded to 138 and 275 times, respectively. The sagittal and tangential rays produced lower MTF values observed on myopic model also indicates the contrast and image sharpness were not at optimum level. It was anticipated earlier from the large tangential and sagittal differences as the image formed is extremely inferior in the mammalian eye study using Guinea pig (Bowrey et al., 2015).

The 2-D layout of the corrected myopic Liou and Brennan eye model from Zemax output is presented in Figure 6. The corrected myopic Liou and Brennan model refracting surfaces are shown accordingly starting from infinity to the anterior cornea, posterior cornea, anterior lens and posterior lens. According to Liou and Brennan model, the retinal curvature was taken into consideration for image formation (de Almeida \& Carvalho, 2007). The concave lens used to correct the myopic refractive error was position in front of the eye at a vertex distance of $15 \mathrm{~mm}$. The vertex distance is a distance from the anterior corneal surface to the back surface of the lens. 


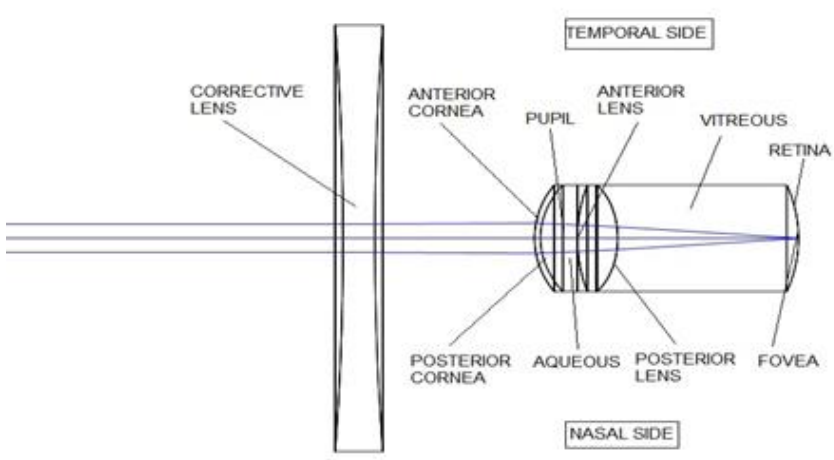

Figure 6. 2-D layout of corrected myopic Liou and Brennan eye model

The tangential and sagittal MTF curves obtained for corrected myopic Liou and Brennan model at visible light wavelength ranging from blue $(486.1 \mathrm{~nm})$ to red $(656.3 \mathrm{~nm})$ is presented in Figure 7. For the corrected myopic Liou and Brennan model, plotting the tangential and sagittal MTF were carried out to illustrate the influence of decentration and tilt of the pupil.

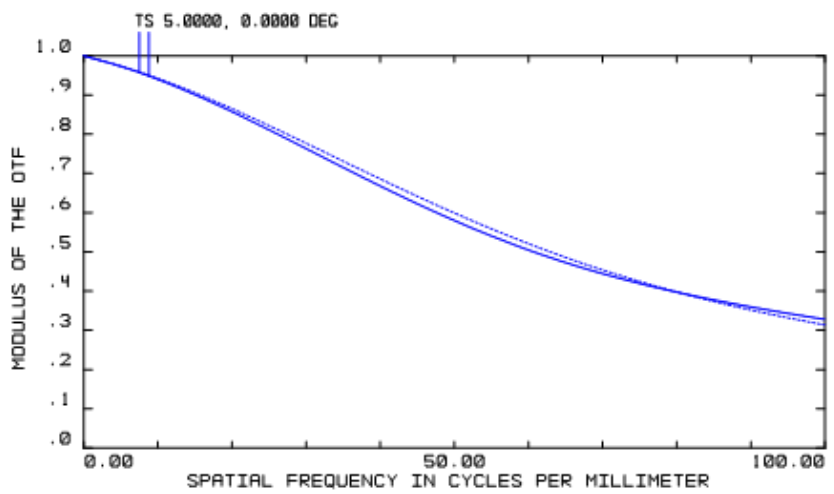

Figure 7. MTF for corrected myopic Liou and Brennan eye model at light wavelength from $486.1 \mathrm{~nm}$ to $656.3 \mathrm{~nm}$.

Tangential ray is denoted by ' $\mathrm{T}$ ' with dash line and sagittal ray is denoted by 'S' with straight line.

The MTF result for corrected myopic Liou and Brennan eye model shown in Figure 7 is separated for tangential and sagittal rays. Also, it shows that the MTF starts from 1 (for perfect) and reduce to lower values with the increment of spatial frequency as the ray approach toward the posterior of the eyeball before reaching the retina.

At spatial frequency of $100 \mathrm{lp} / \mathrm{mm}$, the cut-off of MTF was 0.3138 and 0.3279 for tangential and sagittal, respectively. The MTF result also shows that both tangential and sagittal rays were not overlapping indicate that the model eye is elliptical in shape. It was due to the corrected myopic Liou and Brennan model have multiple refracting surfaces at different distances with different curvatures and refractive indices which caused the ray to be easily diffracted (Atchison \& Smith, 2000).

Further analysis on tangential and sagittal rays, showed that the MTF values of the corrected myopic Liou and Brennan model were higher compared with the previous uncorrected myopic model. At $100 \mathrm{lp} / \mathrm{mm}$ spatial frequency, the MTF for tangential and sagittal ray were $31.38 \%$ and $32.79 \%$, respectively, which was higher than the uncorrected myopic model. However, the corrected myopic model produced lower MTF values for both tangential and sagittal MTF compared with the emmetropic model of Liou and Brennan.

In contrast with emmetropic Liou and Brennan eye, the corrected myopic model was modelled by positioning the corrective lenses in front of the uncorrected myopic eye. The corrective lenses surface curvatures was calculated as described in the Equation (3). Overall, the tangential and sagittal ray were both degraded to 1.5 times from the emmetropic Liou and Brennan model. This degradation was significantly lower compared to the uncorrected myopic model.

In this study, the SER value was used as the corrective lens power. The SER value was calculated as sphere plus the half of the cylindrical component. The astigmatism equal to or less than $0.75 \mathrm{D}$ was not taken into account in providing full correction of the cylindrical component, i.e., the astigmatism. As a result, the MTF values obtained from the corrected model of Liou and Brennan were slightly inferior compared with its emmetropic model.

Comparison of MTF among emmetropic, myopic and corrected myopic of Liou and Brennan eye model are shown in Table 4. The accuracy between the emmetropia and corrected myopia are also presented. Here, the accuracy is calculated as (corrected value using our myopia model calculated using emmetropia model)/ corrected value using our myopia model. The accuracy is calculated to verify the improvement of our myopia model compared to the reported model. 
Table 4. Comparison of MTF among emmetropic, myopic and corrected myopic of Liou and Brennan eye model and its accuracy between emmetropic and corrected myopic (Ttangential ray, S-sagittal ray)

\begin{tabular}{|c|c|c|c|c|c|}
\hline \multirow{2}{*}{$\begin{array}{l}\text { MTF } \\
\text { (\%) }\end{array}$} & \multicolumn{2}{|c|}{ Emmetropia } & \multirow{2}{*}{$\begin{array}{c}\text { Myo- } \\
\text { pia }\end{array}$} & \multirow{2}{*}{$\begin{array}{c}\text { Correc- } \\
\text { ted } \\
\text { Myopia }\end{array}$} & \multirow{2}{*}{$\begin{array}{c}\text { Accu } \\
- \\
\text { racy }\end{array}$} \\
\hline & $\begin{array}{l}\text { Standard } \\
\text { Model }\end{array}$ & Reported & & & \\
\hline $\mathrm{T}$ & 48.420 & $\begin{array}{l}\sim 40.000^{\dagger \dagger} \\
\sim 25.000^{\dagger}\end{array}$ & 0.350 & 31.380 & -0.54 \\
\hline $\mathrm{S}$ & 46.850 & $\begin{array}{l}\sim 40.000^{\dagger \dagger} \\
\sim 30.000^{\dagger}\end{array}$ & 0.170 & 32.790 & -0.43 \\
\hline
\end{tabular}

${ }^{+}$Zoulinakis et al. (2017) using $\lambda=587.6 \mathrm{~nm}$ at EPD of $2 \mathrm{~mm}$ † de Almeida \& Carvalho (2007) using visible white light at EPD of $4 \mathrm{~mm}$

From Table 4, for the MTF value at tangential and sagittal rays at 100 cycles $/ \mathrm{mm}$, the emmetropic Liou and Brennan value was higher than the previous studies (de Almeida \& Carvalho, 2007; Zoulinakis et al., 2017). Whereas the MTF value at tangential and sagittal rays for corrected myopia value was lower compared to the emmetropic version. This results in the accuracy value of tangential and sagittal rays of -0.54 and -0.43 respectively.

\section{CONCLUSION}

From the MTF analysis at 100 cycles/mm, the tangential and sagittal rays of emmetropic Liou and Brennan eye model had the highest optical performance from sharpness ability and contrast behaviour among the myopic and corrected myopic models. As the MTF value indicates the threshold of the image contrast of sinusoidal pattern, the higher the value, the higher image recognition. Whereas the MTF value for myopic Liou and Brennan eye was the lowest compared to the other emmetropic models. Furthermore, the MTF value of the corrected myopic Liou and Brennan model was higher compared to the uncorrected myopic model. However, the corrected myopic model produced lower MTF values for both tangential and sagittal MTF compared with the emmetropic model of Liou and Brennan.

In this study, the accuracy of the MTF for the myopia correction and emmetropia using the Liou and Brennan (1997) model were calculated. It was found that the accuracy of the MTF value for corrected myopia at tangential and sagittal rays was lower.

\section{ACKNOWLEDGEMENT}

This work was partially supported by SEGi Internal Research Fund (Grant no. SEGiIRF/2014-1/FOOPVS-2/15), and the UPM Putra Grants (Grant Nos. GP-IBT/2013/941920o and GP-IPB/2014/9449902).

\section{REFERENCES}

Atchison, DA \& Smith, G 2000, Optics of the human eye, Butterworth-Heinemann, Oxford, UK.

Bowrey, HE, Metse, AP, Leotta, AJ, Zeng, G \& Mcfadden, SA 2015, 'The relationship between image degradation and myopia in the mammalian eye', Clinical and Experimental Optometry, vol. 98, no. 6, pp. 555-563. doi: 10.1111/cxo.12316.

Bullimore, MA, Gilmartin, B, \& Royston, J M 1992, 'Steadystate accommodation and ocular biometry in late-onset myopia', Documenta Ophthalmologica, vol. 8o, no. 2, pp. 143-155. doi: 10.1007/bfoo161240.

Carlson, NB \& Kurtz, D 2016, Clinical procedures for ocular examination, 4th edn, McGraw-Hill, New York, USA.

Chua, SY-L, Sabanayagam, C, Tan, C-S, Lim, LS, Toh, J-Y, Chong, Y-S, Gluckman, PD, Yap, F, Cheng, CY, Ngo, CS,
Wong, TY, Chong, MFF, Saw, SM \& the GUSTO Study Group 2018, 'Diet and risk of myopia in three-year-old Singapore children: the GUSTO cohort', Clinical and Experimental Optometry, vol. 101, no. 5, pp. 692-699. doi: 10.1111/cxo.12677.

De Almeida, MS \& Carvalho, LA 2007, "Different schematic eyes and their accuracy to the in vivo eye: A quantitative comparison study', Brazilian Journal of Physics, vol. 37, no. 2a, pp. 378-387. doi: 10.1590/s010397332007000300008.

Elliot, DB 2014, Clinical procedures in primary eye care, 4th edn, Elsevier/Saunders.

Goss, DA \& West, RW 2002, Introduction to the optics of the eye, Butterworth-Heinemann, Boston, USA. 
Grosvenor, T \& Scott, R 1991, 'Comparison of refractive components in youth-onset and early adult-onset myopia', Optometry and Vision Science, vol. 68, no. 3, pp. 204-209. doi: 10.1097/00006324-199103000-00008.

Gwiazda, J, Wendy, L, Marsh-Tootle, Hyman, L, Hussein, M, Norton, TT \& the COMET Study Group 2002, 'Baseline refractive and ocular component measures of children enrolled in the correction of myopia evaluation trial (COMET)', Investigative Ophthalmology and Visual Science, vol. 43, no. 2, pp. 314-321.

Hiraoka, T, Kotsuka, J, Kakita, T, Okamoto, F \& Oshika, T 2017, 'Relationship between higher-order wavefront aberrations and natural progression of myopia in schoolchildren', Scientific Reports, vol. 7, no. 7876. doi: 10.1038/s41598-017-08177-6.

Jiang, B-C \& Woessner, WM 1996, 'Vitreous chamber elongation is responsible for myopia development in a young adult', Optometry and Vision Science, vol. 73, no. 4, pp. 231-234. doi: 10.1097/00006324-199604000-00003.

Liou, H-L \& Brennan, NA 1997, 'Anatomically accurate, finite model eye for optical modeling', Journal of the Optical Society of America A, vol. 14, no. 8, pp. 1684-1695. doi: 10.1364/josaa.14.001684.

Liu, Y \& Wang, Y 2019, 'Optical quality comparison between laser ablated myopic eyes with centration on coaxially sighted corneal light reflex and on entrance pupil center', Journal of the Optical Society of America A, vol. 36, no. 4, pp. B103. doi: 10.1364/josaa.36.oob103.

McBrien, NA \& Millodot, M 1987, 'A biometric investigation of late onset myopic eyes', Acta Ophthalmologica, vol. 65, pp. 461-468. doi:10.1111/j.1755-3768.1987.tbo7024.x.

Smith, G, Bedggood, P, Ashman, R, Daaboul, M \& Metha, A 2008, 'Exploring ocular aberrations with a schematic human eye model', Optometry and Vision Science, vol. 85, no. 5, pp. 330-340. doi: 10.1097/opx.obo13e31816c4449.

Sun, Y, Xu, F, Zhang, T, Liu, M, Wang, D, Chen, Y \& Liu, Q 2015, 'Correction: Orthokeratology to control myopia progression: A meta-analysis', Plos One, vol. 10, no. 6, pp. e0130646. doi: 10.1371/journal.pone.0130646.

Thibos, LN \& Bradley, A 1999, 'Modelling the refractive and neuro-sensor systems of the eye', eds P Mouroulis, in Visual instrumentation: Optical design and engineering principles, McGraw-Hill, New York, USA, pp. 101-159.

Zadnik, K \& Mutti, DO 2019, 'Outdoor activity protects against childhood myopia - Let the sun shine in', JAMA Pediatrics, vol. 173, no. 5, pp. 415-416. doi: 10.1001/jamapediatrics.2019.0278.
Zapata-Díaz, JF, Radhakrishnan, H, Charman, WN \& LópezGil, N 2019, 'Accommodation and age-dependent eye model based on in vivo measurements', Journal of Optometry, vol. 12, no. 1, pp. 3-13. doi: 10.1016/j.optom.2018.01.003.

Zemax User's Manual 2012, Zemax 12 optical design program, Radiant Zemax LLC, Kirkland, USA.

Zoulinakis, G, Esteve-Taboada, JJ, Ferrer-Blasco, T, MadridCosta, D \& Montés-Micó, R 2017, 'Accommodation in human eye models: A comparison between the optical designs of Navarro, Arizona and Liou-Brennan', International Journal of Ophthalmology, vol. 10, no. 1, pp.43-50. doi:10.18240/ijo.2017.01.07. 